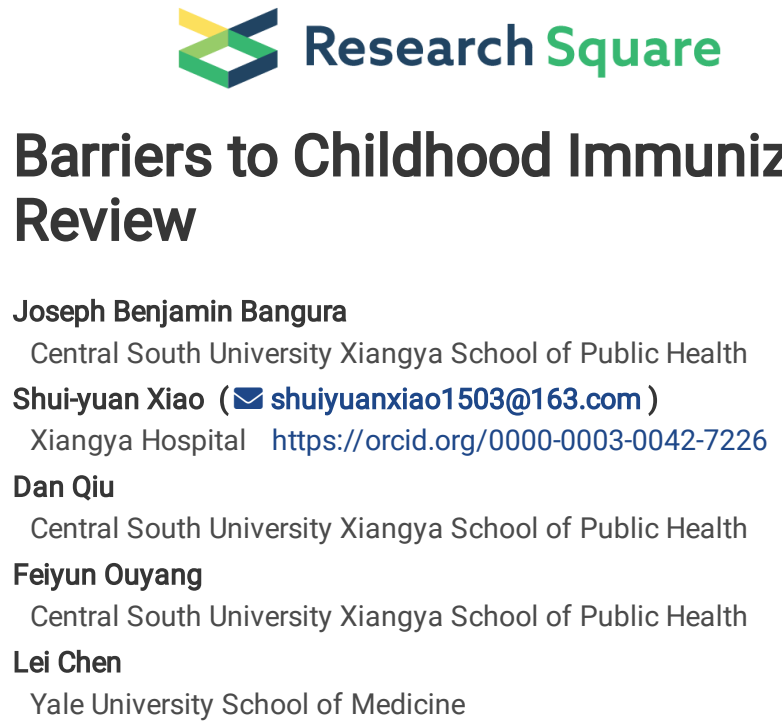

\title{
Barriers to Childhood Immunization in Sub-Saharan Africa: A Systematic Review
}

Version of Record: A version of this preprint was published on July 14th, 2020. See the published version at https://doi.org/10.1186/s12889-020-09169-4. 


\section{Abstract}

Background Immunization to prevent infectious diseases is a core strategy to improve childhood health as well as survival. It remains a challenge for some African countries to attain the required childhood immunization coverage. We aim at identifying individual barriers confronting parents/caretakers, providers, and health systems that hinder childhood immunization coverage in Sub-Saharan Africa.

Method This systematic review searched PubMed/MEDLINE, Web of Science and EMBASE. We restricted to published articles in English that focused on childhood immunization barriers in Sub-Saharan Africa from January 1988 to February 2019. We excluded studies if: focused on barriers to immunization for children in other regions of the world, studied adult immunization barriers; studies not available on the university library, they were editorial, reports, reviews, supplement, and bulletins. Study designs included were cross-sectional, second-hand data analysis; and case control.

Results Of the 2,538 items identified, 40 met inclusion criteria. Parents/caretakers were the most common subjects. Eight articles were of moderate and 32 were of high methodological quality. Seven studies analyzed secondary data; 30 used cross-sectional designs and three employed case control method. Twenty-five studies reported national immunization coverage of key vaccines for children under one, fifteen did not. When reported, national immunization coverages of childhood vaccines were reported to be low. Parents/caretaker's barriers included lack of knowledge of immunization, distance to access point, financial deprivation, lack of partners support, and distrust in vaccines and immunization programs. Other associated factors for low vaccine rates included the number of off-springs, life style, migration and occupation. Barriers at health system level cited by healthcare providers included limited human resources and inadequate infrastructures to maintain the cold chain and adequate supply of vaccines.

Conclusion In this review we identified more thoroughly the parents/caretakers' barriers than those of providers and health systems. Factors that influenced decisions to get children vaccinated were mainly their gender, beliefs, socio-economic and socio-culture factors in the communities in which they live. Thus it is vital that immunization programs consider these barriers and address the people and societies in their communities across SubSaharan Africa.

\section{Background}

Immunization is a protective measure against infectious diseases [1]. Childhood immunization remains one of the most high impact public health interventions, by reducing infectious diseases-related morbidly and mortality of children at a low cost [2]. It is a core child survival strategy and is demonstrated to avert more than 1.2 million child deaths each year [3, 4]. It is a key strategy towards attaining Sustainable Development Goal number 3 , namely the reduction of under-five mortality to less than 25/1000 live births by 2013 [5]. Despite these gains, vaccine-preventable diseases remain a major cause of child illnesses and deaths, particularly in low-income countries [6].

Africa has the highest under-five mortality rate of the entire world and accounts for $40 \%$ of the total deaths in this age group. This is mainly due to vaccine-preventable diseases [7]. Over the past few decades, African immunization programs have made progress, yet coverages remain low for some recommended childhood vaccines. In 2014, it was reported that only Zimbabwe among the Sub-Saharan region was estimated to have met the Global Vaccine Action Plan threshold of $80 \%$ or higher coverage of diphtheria-tetanus-pertussis vaccine (DTP3), a benchmark used to measure performance of routine vaccine delivery system [8]. In 2016, one in five African children goes without lifesaving vaccines [9]. Most African countries are unable to reach the most vulnerable children populations in remote and rural communities $[5,10]$. Studies $[1-3,7,11-54]$ conducted in Africa have attempted to elucidate potential barriers that lead to low uptake and none-completion of immunization series. Previous review [55] exploring reasons related to non-vaccination and under-vaccination of children in low and middle income countries categorized factors into major themes: Immunization systems; communication and information; family characteristics and parental attitudes/knowledge. However, it noted the lack of peer reviewed literature in Central Africa. Another review [56] investigated factors associated with incomplete or delayed vaccination across countries. Despite its potential importance, it did not categorized findings into major domains, as policy implication for each might be different.

This systematic review aims at identifying relevant studies and summarizing major barriers confronting health systems, providers, and caregivers that hinder immunization coverage in Sub-Saharan Africa. The results of this review will add to existing knowledge of the problem, and guide policy makers to improve immunization programs in Sub-Saharan Africa, especially in those countries where the included studies were conducted; and also to provide useful information for further research on these problems.

\section{Methods}

\section{Search strategy and Study selection}

The study employed Preferred Reporting Items for Systematic Reviews and Meta- Analysis (PRSMA) guidelines [57]. We performed electronic searches of articles included in this systematic review from the Web of Science, PubMed/Medline and EMBASE from January, 1988 to December, 2019 . We combined the following terms: ((child or children or childhood or infant or baby or newborn) and (immunization or immunisation or vaccination or vaccine or immunity) and (barrier or hesitant or refuse or refusal or delay or denial or denier or denied or concern or reason or doubt "non-acceptance" or incomplete or obstacle or constraint) and ("Sub Saharan Africa" or Angola or Benin or Botswana or "Burkina Faso" or Burundi or "Cabo Verde" or Cameroon or "Central African Republic" or Chad or Comoros or Congo or "Cote d'Ivoire" or "Equatorial Guinea” or Eritrea or Ethiopia or Gabon or Gambia or Ghana or Guinea or "Guinea- Bissau” or Kenya or Lesotho or Liberia or Madagascar or Malawi or Mali or Mauritania or Mauritius or Mozambique or Namibia or 
Niger or Nigeria or Rwanda or Senegal or Sierra Leone or Somalia or "South Africa" or "South Sudan" or Sudan or Tanzania or Togo or Uganda or Zambia or Zimbabwe)). [See supplementary materials 1]

We restricted to published articles in English that focused on childhood immunization barriers, conducted in Sub-Saharan Africa from January 1988 to December 2019. Articles were excluded if: (i) focused on barriers to immunization for children in other regions of the world, (ii) studied adult immunization barriers (iii) published before December, 1988 and beyond December, 2019; (iv) they were editorials, (v) reports, (vi) review articles, (vii) supplement articles, (vii) bulletins and (ix) studies not available on the university library. We included only observational studies in this systematic review. This review was registered on ORCiD Registry with ID: 0000-0003-0042-7226

\section{Data analysis}

Search result items were uploaded into EndNote X7 library. Duplicate were removed. JBB and DQ did the initial screening (title and abstract) and full texts of articles based on the inclusion and exclusion criteria. We resolved disagreements with third review (FO). The study employed narrative synthesis. We systematically compare authors' different perspectives, studies' methodology, and findings. We develop a preliminary synthesis. The authors employed the following approaches: tabulation and thematic analysis. For each included study, the following data were extracted: Author, year, geographical location and number of countries included in a study; participants and demographic; study design; reported national immunization coverage, data source, date; study quality; and key reported barriers. We organized this synthesis into parental barriers; health system barriers and provider's barriers. JBB synthesized data and created table with input from LC and SX. Classifications were performed by JBB and LC. Discrepancies were resolved by consensus after discussions.

\section{Study Methodological Quality}

JBB and DQ assessed articles for methodological quality independently based on modified tool designed to assess quantitative and qualitative studies used in a similar study published elsewhere [58]. [See supplementary materials 2] It included a range of items from 1 to 14 . Each item scores one point. Based on the scores, we grouped articles into three: low, moderate and high; articles scored 12 points and above were considered high methodological quality, moderate 8 to11 points,7 points and below were low. If ratings differed, we discussed the article in an effort to arrive consensus.

\section{Results}

Our database searches yielded 2,652 records. 2,250 records were screened by title and abstract after duplicates removed; 76 full-text articles assessed for eligibility. Forty-eight articles met all inclusion criteria for this study; 28 articles were excluded for various reasons. [See supplementary materials 3 ] Nine articles employed second-hand data analysis [7, 12, 18, 27, 37, 40, 41, 47, 42]; thirty-six used cross-sectional design [1-3, 11, 13-17, 19, 21-26, 28-36, $38-39,42,45-46,48-51,53,54]$ and three used case-control study [20, 43-44]. All described studies were conducted on Africa populations (103,655 adults and 76,327 children). Forty-seven articles focus on identifying barriers to general childhood vaccination 0-59 months [1-3, 7, 11-17, 19-54] in the following countries: Uganda, Ethiopia, Kenya, Sudan, Nigeria, Gabon, Cameroon, South Africa, Tanzania, Burkina Faso, Togo, Ghana, Malawi and few unspecified countries in Sub-Saharan. One critically examined barriers specific to vaccination doses at birth 0-1 day [18] in the Gambia. Thirty articles reported national immunization coverages [1-2, 7, 11-16, 20-25, 28-29, 32, 34-35, 38-40, 43-46, 49, 50, 52-54], eighteen did not [3, 17-19, 22, 24, $26-27,30-31,33,36-37,41-42,47,48,51] .39$ articles were classed as high and 9 were moderate methodological quality. We split results into three sections - parental barriers, health system barriers, and providers' barriers. 8 studies examined all - parental/caretakers, health systems and providers' barriers $[3,11,13,16,43,45,48,52]$; 21 studies examined parental/caretakers and health system barriers [1, 2, 14, 15, 18-19, 22-23, 25, 29, 31-33, 35, $38,44,47,49-51,53] ; 18$ studies only looked at parental//caretakers barriers [7, 12, 17, 20-21, 24, 26-28, 34, 36-37, 39, 42, 46, 54] and one study examined parental and providers' barriers. Several Sub-Saharan countries were reported to have low childhood immunization coverages with variations across the region. Nigeria reported lowest with $12 \cdot 7 \%$ in 2013 and Ethiopia highest with $88 \%$ in 2013 . See summary table for studies' characteristics and key findings. 
Table 1

Summary of studies' characteristics and key findings

\begin{tabular}{|c|c|c|c|c|c|c|c|}
\hline \multirow{2}{*}{$\begin{array}{l}\text { Author, year, } \\
\text { geographical } \\
\text { location and } \\
\text { number of } \\
\text { countries } \\
\text { included in } \\
\text { the study }\end{array}$} & \multirow{2}{*}{$\begin{array}{l}\text { Participants and } \\
\text { demographic }\end{array}$} & \multirow{2}{*}{$\begin{array}{l}\text { Study } \\
\text { design }\end{array}$} & \multirow{2}{*}{$\begin{array}{l}\text { Reported national } \\
\text { immunization } \\
\text { coverage, Data } \\
\text { source, Date }\end{array}$} & \multirow[t]{2}{*}{ Quality } & \multicolumn{3}{|c|}{ Key childhood immunization reported barriers } \\
\hline & & & & & $\begin{array}{l}\text { Parental/caretakers } \\
\text { barriers }\end{array}$ & $\begin{array}{l}\text { Health systems } \\
\text { barriers }\end{array}$ & $\begin{array}{l}\text { Providers } \\
\text { barriers }\end{array}$ \\
\hline $\begin{array}{l}\text { Tadesse et al } \\
{[1],} \\
2009 \\
\text { Ethiopia } \\
\text { East Africa, } 1\end{array}$ & 126 adults & $\begin{array}{l}\text { Cross- } \\
\text { sectional }\end{array}$ & $\begin{array}{l}38 \cdot 5 \% \text { National } \\
\text { health survey of } \\
\text { Ethiopia, } 2006\end{array}$ & Moderate & $\begin{array}{l}\text { Misunderstanding } \\
\text { of side effects, } \\
\text { busy with seasonal } \\
\text { farm work }\end{array}$ & $\begin{array}{l}\text { Absence of } \\
\text { electricity to } \\
\text { maintain the } \\
\text { cold chain }\end{array}$ & \\
\hline $\begin{array}{l}\text { Obasoha et } \\
\text { al [2], } \\
2018 \\
\text { Nigeria } \\
\text { West Africa, } \\
1\end{array}$ & 215 mothers & $\begin{array}{l}\text { Cross- } \\
\text { sectional }\end{array}$ & $\begin{array}{l}\text { 25\%, World Health } \\
\text { Organization, Global } \\
\text { Immunization } \\
\text { Vision and } \\
\text { Strategies, } 2013\end{array}$ & High & $\begin{array}{l}\text { Unaware of the } \\
\text { need of } \\
\text { immunization, lack } \\
\text { of information, fear } \\
\text { of side effect }\end{array}$ & $\begin{array}{l}\text { Vaccines not } \\
\text { available, } \\
\text { vaccinators } \\
\text { absence long } \\
\text { distance to } \\
\text { cover }\end{array}$ & \\
\hline $\begin{array}{l}\text { Malande et } \\
\text { al [3], } \\
2014 \\
\text { Uganda } \\
\text { East Africa, } 1\end{array}$ & $\begin{array}{l}311 \\
\text { caretakers/child } \\
\text { pairs }\end{array}$ & $\begin{array}{l}\text { Cross - } \\
\text { sectional }\end{array}$ & Not reported & High & $\begin{array}{l}\text { transport } \\
\text { difficulties, difficult } \\
\text { geographical } \\
\text { terrain, language } \\
\text { barrier, less support } \\
\text { from husband }\end{array}$ & $\begin{array}{l}\text { Vaccine stock } \\
\text { outs, difficult } \\
\text { terrain and poor } \\
\text { road network, } \\
\text { inadequate } \\
\text { transportation } \\
\text { means, poor } \\
\text { working } \\
\text { condition of } \\
\text { vaccine fridges, } \\
\text { inadequate } \\
\text { staff, long } \\
\text { distance }\end{array}$ & $\begin{array}{l}\text { Lack of } \\
\text { knowledge of } \\
\text { vaccines } \\
\text { adverse effects }\end{array}$ \\
\hline $\begin{array}{l}\text { Wiysonge et } \\
\text { al [7], } \\
2012 \\
\text { Sub-Saharan } \\
\text { Africa } \\
\text { countries, } 24\end{array}$ & $\begin{array}{l}27,094 \text { children } \\
\text { aged } 12-23 \text { month }\end{array}$ & $\begin{array}{l}\text { Second- } \\
\text { hand data } \\
\text { Analysis }\end{array}$ & $\begin{array}{l}71 \% \text {, WHO (2010); } \\
\text { vaccine preventable } \\
\text { diseases: } \\
\text { monitoring syatem- } \\
2010\end{array}$ & High & $\begin{array}{l}\text { poor households, } \\
\text { high illiteracy rates, } \\
\text { lack of vaccines } \\
\text { information, poor } \\
\text { health seeking } \\
\text { behaviors (Not } \\
\text { going for ANC visit) }\end{array}$ & & \\
\hline $\begin{array}{l}\text { Tefera et al } \\
\text { [11], } \\
2018 \\
\text { Ethiopia } \\
\text { East Africa, } 1\end{array}$ & $\begin{array}{l}540 \text { mothers with } \\
\text { children aged } \\
\text { between } 12 \text { and } 23 \\
\text { months }\end{array}$ & $\begin{array}{l}\text { Cross - } \\
\text { sectional }\end{array}$ & $\begin{array}{l}86 \%, \text { WHO/UNICEF } \\
\text { Immunization } \\
\text { coverage ,2015 }\end{array}$ & High & $\begin{array}{l}\text { fear of side } \\
\text { reactions, lack of } \\
\text { information, being } \\
\text { too busy, } \\
\text { place/time being } \\
\text { unknown, long } \\
\text { waiting time, }\end{array}$ & $\begin{array}{l}\text { vaccinators } \\
\text { were absent, } \\
\text { vaccines were } \\
\text { not available, } \\
\text { limited health } \\
\text { facilities, } \\
\text { vaccine site } \\
\text { being too far }\end{array}$ & \\
\hline $\begin{array}{l}\text { Porth et al } \\
\text { [12], } 2019 \\
\text { Ethiopia } \\
\text { East Africa, } 1\end{array}$ & 2,722 children & $\begin{array}{l}\text { Second- } \\
\text { hand data } \\
\text { Analysis }\end{array}$ & $\begin{array}{l}39 \% \text {, Ethiopia } \\
\text { Demographic and } \\
\text { health survey, } 2016\end{array}$ & High & $\begin{array}{l}\text { Negative } \\
\text { perception of } \\
\text { vaccines, religion, } \\
\text { waiting too long }\end{array}$ & $\begin{array}{l}\text { limited } \\
\text { operating hours, } \\
\text { clinic distance }\end{array}$ & \\
\hline $\begin{array}{l}\text { Kiptoo et al } \\
{[13],} \\
2015 \\
\text { Kenya } \\
\text { East Africa, } 1\end{array}$ & $\begin{array}{l}298 \\
\text { mothers/guardians }\end{array}$ & $\begin{array}{l}\text { Cross - } \\
\text { sectional }\end{array}$ & $\begin{array}{l}86 \%, \text { Kenya } \\
\text { Expanded program } \\
\text { on immunization, } \\
2009\end{array}$ & High & $\begin{array}{l}\text { lack of knowledge, } \\
\text { earning less, many } \\
\text { siblings }\end{array}$ & $\begin{array}{l}\text { Inadequate } \\
\text { health facilities, } \\
\text { Long distance } \\
\text { for out-reach } \\
\text { services }\end{array}$ & \\
\hline $\begin{array}{l}\text { Cockcroft et } \\
\text { al [14], } \\
2014 \\
\text { Nigeria } \\
\text { West Africa, } \\
1\end{array}$ & 2,836 children & $\begin{array}{l}\text { Cross- } \\
\text { sectional }\end{array}$ & $\begin{array}{l}42 \% \text {, Nigeria } \\
\text { Demographic and } \\
\text { Health Survey, } 2013\end{array}$ & High & $\begin{array}{l}\text { Misconception } \\
\text { about vaccines, } \\
\text { fear of side effect, } \\
\text { negligence, being } \\
\text { busy with other } \\
\text { household work }\end{array}$ & $\begin{array}{l}\text { Lack of } \\
\text { vaccines }\end{array}$ & \\
\hline $\begin{array}{l}\text { Nolna et al } \\
\text { [15], } 2018 \\
\text { Cameroon } \\
\text { West Africa, } \\
1\end{array}$ & 1,134 caretakers & $\begin{array}{l}\text { Cross- } \\
\text { sectional }\end{array}$ & $\begin{array}{l}82 \% \text {, WHO-UNICEF } \\
\text { estimates of DPT3 } \\
\text { coverage, } 2017\end{array}$ & High & $\begin{array}{l}\text { Lack of money, } \\
\text { lack of knowledge } \\
\text { of vaccines } \\
\text { importance, busy } \\
\text { with other seasonal } \\
\text { work }\end{array}$ & $\begin{array}{l}\text { Shortage of } \\
\text { health } \\
\text { personnel, } \\
\text { inadequate } \\
\text { means of } \\
\text { transportation }\end{array}$ & \\
\hline
\end{tabular}




\begin{tabular}{|c|c|c|c|c|c|c|c|}
\hline $\begin{array}{l}\text { Author, year, } \\
\text { geographical } \\
\text { location and } \\
\text { number of } \\
\text { countries } \\
\text { included in } \\
\text { the study }\end{array}$ & $\begin{array}{l}\text { Participants and } \\
\text { demographic }\end{array}$ & $\begin{array}{l}\text { Study } \\
\text { design }\end{array}$ & $\begin{array}{l}\text { Reported national } \\
\text { immunization } \\
\text { coverage, Data } \\
\text { source, Date }\end{array}$ & Quality & Key childhood immu & ization reported b & iers \\
\hline $\begin{array}{l}\text { Zewdie et al } \\
\text { [16], } 2016 \\
\text { Ethiopia } \\
\text { East Africa, } 1\end{array}$ & 28 mothers & $\begin{array}{l}\text { Cross- } \\
\text { sectional }\end{array}$ & $\begin{array}{l}88 \% \text {, Ethiopia } \\
\text { National } \\
\text { immunization } \\
\text { survey, } 2013\end{array}$ & Moderate & $\begin{array}{l}\text { Lack of } \\
\text { information, lack of } \\
\text { support from male } \\
\text { partners, high } \\
\text { workload, fear of } \\
\text { mistreatment and } \\
\text { lack of cooperation } \\
\text { from service } \\
\text { providers }\end{array}$ & $\begin{array}{l}\text { Poor } \\
\text { arrangement } \\
\text { and } \\
\text { coordination of } \\
\text { immunization } \\
\text { services, } \\
\text { vaccines stock } \\
\text { out, lack of } \\
\text { viable defaulter } \\
\text { tracking system }\end{array}$ & $\begin{array}{l}\text { Inadequate } \\
\text { home visit, lack } \\
\text { of } \\
\text { commitment, } \\
\text { poor } \\
\text { counseling } \\
\text { skills }\end{array}$ \\
\hline $\begin{array}{l}\text { Babirye et al } \\
{[17],} \\
2011 \\
\text { Uganda } \\
\text { East Africa, } 1\end{array}$ & 1000 adults & $\begin{array}{l}\text { Cross- } \\
\text { sectional }\end{array}$ & Not reported & High & $\begin{array}{l}\text { male partner non- } \\
\text { supportive, Lack of } \\
\text { clothing, money for } \\
\text { transport, or time, } \\
\text { lack of trust in } \\
\text { immunization, fear } \\
\text { of associated side } \\
\text { effects, less } \\
\text { education }\end{array}$ & & \\
\hline $\begin{array}{l}\text { Miyahara et } \\
\text { al [18], } \\
2016 \\
\text { Gambia } \\
\text { West Africa, } \\
1\end{array}$ & $\begin{array}{l}50,455 \text { residents } \\
\text { including children }\end{array}$ & $\begin{array}{l}\text { Second- } \\
\text { hand data } \\
\text { Analysis }\end{array}$ & Not reported & High & $\begin{array}{l}\text { living in urban and } \\
\text { peri-urban settings, } \\
\text { long distance, } \\
\text { ethnicity, low } \\
\text { maternal } \\
\text { education, life style }\end{array}$ & $\begin{array}{l}\text { use of multi- } \\
\text { dose vails with } \\
\text { limited time }\end{array}$ & \\
\hline $\begin{array}{l}\text { Pertet et al } \\
\text { [19], } \\
2018 \\
\text { Kenya } \\
\text { East Africa, } 1\end{array}$ & 515 mothers & $\begin{array}{l}\text { Cross- } \\
\text { sectional }\end{array}$ & Not reported & High & $\begin{array}{l}\text { Movement of the } \\
\text { whole family } \\
\text { (migration), difficult } \\
\text { to access the } \\
\text { health facility due } \\
\text { to bad terrines }\end{array}$ & $\begin{array}{l}\text { Lack of } \\
\text { vaccines }\end{array}$ & \\
\hline $\begin{array}{l}\text { Yenit et al } \\
\text { [20], 2018 } \\
\text { Ethiopia } \\
\text { East Africa, } 1\end{array}$ & 308 mothers & $\begin{array}{l}\text { Case- } \\
\text { control } \\
\text { study }\end{array}$ & $\begin{array}{l}\text { 39\%, Ethiopian } \\
\text { Demographic and } \\
\text { Health Survey, } 2016\end{array}$ & High & $\begin{array}{l}\text { Delivery at home, } \\
\text { lack of antenatal } \\
\text { and postnatal care } \\
\text { visit, miss } \\
\text { conception about } \\
\text { vaccines, }\end{array}$ & & \\
\hline $\begin{array}{l}\text { Tugumisirize } \\
\text { et al [21], } \\
2002 \\
\text { Uganda } \\
\text { East Africa, } 1\end{array}$ & 408 caretakers & $\begin{array}{l}\text { Cross- } \\
\text { sectional }\end{array}$ & $\begin{array}{l}\text { 29\%, Uganda } \\
\text { Demographic and } \\
\text { Health Survey, } 1995\end{array}$ & High & $\begin{array}{l}\text { Fear of rude health } \\
\text { workers, being } \\
\text { busy, low level of } \\
\text { formal education, } \\
\text { fear of side effects, } \\
\text { perceived } \\
\text { contradictions, }\end{array}$ & long distance & \\
\hline $\begin{array}{l}\text { Babalola S. } \\
\text { [22], } 2011 \\
\text { Nigeria } \\
\text { West Africa, } \\
1\end{array}$ & 882 women & $\begin{array}{l}\text { Cross- } \\
\text { sectional }\end{array}$ & Not reported & High & $\begin{array}{l}\text { Lack of knowledge } \\
\text { about } \\
\text { immunization } \\
\text { schedule and } \\
\text { sources, spouses } \\
\text { disapproval of } \\
\text { immunization, } \\
\text { myth and rumors } \\
\text { about side effects, } \\
\text { mothers too busy, } \\
\text { religious and } \\
\text { cultural beliefs, } \\
\text { home delivery }\end{array}$ & $\begin{array}{l}\text { Vaccines } \\
\text { unavailability, } \\
\text { long distance }\end{array}$ & \\
\hline $\begin{array}{l}\text { Oladokun et } \\
\text { al [23], } \\
2010 \\
\text { Nigeria } \\
\text { West Africa, } \\
1\end{array}$ & 248 mothers & $\begin{array}{l}\text { Cross- } \\
\text { sectional }\end{array}$ & $\begin{array}{l}12 \cdot 7 \% \text { National } \\
\text { immunization } \\
\text { survey, } 2003\end{array}$ & High & $\begin{array}{l}\text { Religion, low } \\
\text { mother's education, } \\
\text { mothers not being } \\
\text { aware of additional } \\
\text { doses }\end{array}$ & $\begin{array}{l}\text { Non-availability } \\
\text { of vaccines, }\end{array}$ & \\
\hline
\end{tabular}




\begin{tabular}{|c|c|c|c|c|c|c|c|}
\hline $\begin{array}{l}\text { Author, year, } \\
\text { geographical } \\
\text { location and } \\
\text { number of } \\
\text { countries } \\
\text { included in } \\
\text { the study }\end{array}$ & $\begin{array}{l}\text { Participants and } \\
\text { demographic }\end{array}$ & $\begin{array}{l}\text { Study } \\
\text { design }\end{array}$ & $\begin{array}{l}\text { Reported national } \\
\text { immunization } \\
\text { coverage, Data } \\
\text { source, Date }\end{array}$ & Quality & Key childhood immu & ization reported ba & iers \\
\hline $\begin{array}{l}\text { Schwarz et al } \\
{[24]} \\
2009 \\
\text { Gabon } \\
\text { West Africa, } \\
1\end{array}$ & 262 mothers & $\begin{array}{l}\text { Cross- } \\
\text { sectional }\end{array}$ & Not reported & Moderate & $\begin{array}{l}\text { Transport cost, } \\
\text { feeling ashamed of } \\
\text { poverty-associated } \\
\text { reasons such as } \\
\text { poorly cloth child or } \\
\text { dirty, lack of } \\
\text { knowledge, }\end{array}$ & Long distance & \\
\hline $\begin{array}{l}\text { Ismail et al } \\
{[25],} \\
2014 \\
\text { Sudan } \\
\text { East Africa, } 1\end{array}$ & 213 children & $\begin{array}{l}\text { Cross- } \\
\text { sectional }\end{array}$ & $\begin{array}{l}60 \% \text {, Federal } \\
\text { Ministry of Health, } \\
2005\end{array}$ & High & $\begin{array}{l}\text { lack of knowledge, } \\
\text { long distance, } \\
\text { mothers too busy, } \\
\text { many siblings, fear } \\
\text { of side effect }\end{array}$ & $\begin{array}{l}\text { Vaccinators } \\
\text { absence, } \\
\text { vaccine stock } \\
\text { out }\end{array}$ & \\
\hline $\begin{array}{l}\text { Rees et al } \\
\text { [26], } 1991 \\
\text { South Africa } \\
\text { South Africa, } \\
1\end{array}$ & 315 women & $\begin{array}{l}\text { Cross- } \\
\text { sectional }\end{array}$ & Not reported & High & $\begin{array}{l}\text { low literacy level of } \\
\text { mothers }\end{array}$ & $\begin{array}{l}\text { Long distance } \\
\text { to reach the } \\
\text { facility }\end{array}$ & \\
\hline $\begin{array}{l}\text { Nadella et al } \\
\text { [27], } 2019 \\
\text { Tanzania } \\
\text { East Africa, } 1\end{array}$ & 31,999 children & $\begin{array}{l}\text { Secondary } \\
\text { data } \\
\text { analysis }\end{array}$ & Not reported & High & $\begin{array}{l}\text { Parents not } \\
\text { educated, mothers } \\
\text { not attending ANC, } \\
\text { delivery at home, } \\
\text { poor household }\end{array}$ & & \\
\hline $\begin{array}{l}\text { Meleko et al } \\
\text { [28], 2017 } \\
\text { Ethiopia } \\
\text { East Africa, } 1\end{array}$ & $\begin{array}{l}322 \\
\text { mothers/caretakers }\end{array}$ & $\begin{array}{l}\text { cross- } \\
\text { sectional }\end{array}$ & $\begin{array}{l}\text { 24.3\% Ethiopia } \\
\text { Demographic } \\
\text { Health Survey } \\
\text { (EDHS), } 2011\end{array}$ & High & $\begin{array}{l}\text { Low parental } \\
\text { educational level, } \\
\text { delivery at home, } \\
\text { parents not } \\
\text { utilizing maternal } \\
\text { health care } \\
\text { services. Lack of } \\
\text { knowledge }\end{array}$ & & \\
\hline $\begin{array}{l}\text { Itimi et al } \\
\text { [29], } 2012 \\
\text { Nigeria } \\
\text { west Africa, } 1\end{array}$ & 558 women & $\begin{array}{l}\text { cross- } \\
\text { sectional }\end{array}$ & $\begin{array}{l}\text { 23\% Nigeria } \\
\text { Demographic and } \\
\text { Health Survey, } 2008\end{array}$ & High & $\begin{array}{l}\text { Adverse rumor } \\
\text { about childhood } \\
\text { immunization }\end{array}$ & $\begin{array}{l}\text { Inadequate } \\
\text { health personnel } \\
\text { (Vaccinators) }\end{array}$ & \\
\hline $\begin{array}{l}\text { Kagoné et al } \\
{[30], 2018} \\
\text { Burkina Faso } \\
\text { West Africa, } \\
1\end{array}$ & Not stated & $\begin{array}{l}\text { cross- } \\
\text { sectional }\end{array}$ & Not reported & Moderate & $\begin{array}{l}\text { Migration, mothers } \\
\text { being busy, poor } \\
\text { interaction between } \\
\text { women and health } \\
\text { workers, potential } \\
\text { adverse events, } \\
\text { geographic (Hard } \\
\text { to reach arears), } \\
\text { and lack of } \\
\text { information. }\end{array}$ & & $\begin{array}{l}\text { Not open multi- } \\
\text { dose vails } \\
\text { unless a critical } \\
\text { number of } \\
\text { children are } \\
\text { present }\end{array}$ \\
\hline $\begin{array}{l}\text { Tobin-West } \\
\text { et al [31], } \\
2012 \\
\text { Nigeria } \\
\text { West Africa, } \\
1\end{array}$ & $\begin{array}{l}1560 \\
\text { mothers/caregivers }\end{array}$ & $\begin{array}{l}\text { cross- } \\
\text { sectional }\end{array}$ & Not reported & High & $\begin{array}{l}\text { Long waiting time, } \\
\text { belief in the } \\
\text { efficacy of } \\
\text { traditional } \\
\text { medicines as an } \\
\text { alternative to } \\
\text { immunization, poor } \\
\text { rapport with health } \\
\text { workers, }\end{array}$ & $\begin{array}{l}\text { Frequent } \\
\text { shortage of } \\
\text { vaccine }\end{array}$ & \\
\hline $\begin{array}{l}\text { Braka et al } \\
\text { [32], } 2011 \\
\text { Uganda } \\
\text { East Africa, } 1\end{array}$ & 136 caretakers & $\begin{array}{l}\text { cross- } \\
\text { sectional }\end{array}$ & $\begin{array}{l}80 \% \text { W H O } \\
\text { Immunization } \\
\text { profile-Uganda } \\
1980-2008,2010\end{array}$ & High & $\begin{array}{l}\text { Misconceptions, } \\
\text { adverse effects } \\
\text { experience, } \\
\text { providers' bad } \\
\text { attitudes }\end{array}$ & $\begin{array}{l}\text { Inadequate staff } \\
\text { at health center } \\
\text { level, poor } \\
\text { storage facilities } \\
\text { for vaccines, }\end{array}$ & \\
\hline $\begin{array}{l}\text { Ambe et al } \\
\text { [33], } 2001 \\
\text { Nigeria } \\
\text { West Africa, } \\
1\end{array}$ & 500 mothers & $\begin{array}{l}\text { cross- } \\
\text { sectional }\end{array}$ & Not reported & High & $\begin{array}{l}\text { Parents don't have } \\
\text { trust in vaccines, } \\
\text { parents are abused } \\
\text { in hospitals, } \\
\text { husband } \\
\text { refused/not } \\
\text { supportive }\end{array}$ & $\begin{array}{l}\text { Vaccines not } \\
\text { available }\end{array}$ & \\
\hline
\end{tabular}




\begin{tabular}{|c|c|c|c|c|c|c|}
\hline $\begin{array}{l}\text { Author, year, } \\
\text { geographical } \\
\text { location and } \\
\text { number of } \\
\text { countries } \\
\text { included in } \\
\text { the study }\end{array}$ & $\begin{array}{l}\text { Participants and } \\
\text { demographic }\end{array}$ & $\begin{array}{l}\text { Study } \\
\text { design }\end{array}$ & $\begin{array}{l}\text { Reported national } \\
\text { immunization } \\
\text { coverage, Data } \\
\text { source, Date }\end{array}$ & Quality & Key childhood immu & ization reported barriers \\
\hline $\begin{array}{l}\text { Tadesse et al } \\
\text { [34], 2009 } \\
\text { Ethiopia } \\
\text { East Africa, } 1\end{array}$ & 226 children & $\begin{array}{l}\text { cross- } \\
\text { sectional }\end{array}$ & $\begin{array}{l}49.9 \% \text { Federal } \\
\text { Ministry of Health } \\
\text { Ethiopia, } 2006\end{array}$ & High & $\begin{array}{l}\text { Poor knowledge } \\
\text { about } \\
\text { immunization, } \\
\text { mother's negative } \\
\text { perceptions, low } \\
\text { monthly income of } \\
\text { parents }\end{array}$ & \\
\hline $\begin{array}{l}\text { Jani et al } \\
{[35], 2008} \\
\text { Mozambique } \\
\text { South Africa, } \\
1\end{array}$ & 668 mothers & $\begin{array}{l}\text { cross- } \\
\text { sectional }\end{array}$ & $\begin{array}{l}80 \% \text { Expanded } \\
\text { program on } \\
\text { immunization, } \\
1998\end{array}$ & High & $\begin{array}{l}\text { Low education } \\
\text { level of mothers, } \\
\text { long waiting time } \\
\text { for vaccination, } \\
\text { parent's } \\
\text { forgetfulness, } \\
\text { migration, } \\
\text { concomitant } \\
\text { treatment by } \\
\text { traditional healers }\end{array}$ & $\begin{array}{l}\text { Vaccines } \\
\text { shortage, } \\
\text { Inadequate } \\
\text { health workers } \\
\text { (Vaccinators) }\end{array}$ \\
\hline $\begin{array}{l}\text { Eng et al [36], } \\
1991 \\
\text { Togo } \\
\text { West Africa, } \\
1\end{array}$ & $\begin{array}{l}110 \\
\text { mothers/caretakers }\end{array}$ & $\begin{array}{l}\text { cross- } \\
\text { sectional }\end{array}$ & Not reported & Moderate & $\begin{array}{l}\text { Lack of knowledge, } \\
\text { parent's } \\
\text { forgetfulness, } \\
\text { health workers } \\
\text { being aggressive, } \\
\text { long waiting time, } \\
\text { laziness, lack of } \\
\text { information about } \\
\text { vaccines, low } \\
\text { income of parents }\end{array}$ & $\begin{array}{l}\text { Long distance } \\
\text { to health } \\
\text { facilities }\end{array}$ \\
\hline $\begin{array}{l}\text { Landoh et al } \\
\text { [37], } 2016 \\
\text { Togo } \\
\text { West Africa, } \\
1\end{array}$ & $\begin{array}{l}2067 \text { children } \\
\text { (12 to } 59 \text { months) }\end{array}$ & $\begin{array}{l}\text { Secondary } \\
\text { data } \\
\text { analysis }\end{array}$ & Not reported & High & $\begin{array}{l}\text { Residence of } \\
\text { mother (Muslims } \\
\text { dominated), non- } \\
\text { schooled mothers, } \\
\text { being a single } \\
\text { mother, negative } \\
\text { cultural beliefs }\end{array}$ & \\
\hline $\begin{array}{l}\text { Legesse et al } \\
\text { [38], } 2015 \\
\text { Ethiopia } \\
\text { East Africa, } 1\end{array}$ & $\begin{array}{l}591 \text { children } 12 \text { to } \\
23 \text { months and } \\
\text { their mothers }\end{array}$ & $\begin{array}{l}\text { cross- } \\
\text { sectional }\end{array}$ & $\begin{array}{l}\text { 36.5\% Ethiopia } \\
\text { Demographic } \\
\text { Health Survey } \\
\text { (EDHS), } 2011\end{array}$ & High & $\begin{array}{l}\text { Lack of knowledge, } \\
\text { lack of information, } \\
\text { low family income, } \\
\text { low education level } \\
\text { of parents, low } \\
\text { maternal health } \\
\text { care utilization, fear } \\
\text { of adverse } \\
\text { reactions, lack of } \\
\text { trust on } \\
\text { immunization, } \\
\text { male partners non } \\
\text { supportive }\end{array}$ & $\begin{array}{l}\text { Poor quality of } \\
\text { health } \\
\text { information } \\
\text { regarding } \\
\text { immunization, } \\
\text { long distance to } \\
\text { health facilities, }\end{array}$ \\
\hline $\begin{array}{l}\text { Wemakor et } \\
\text { al [39], } 2018 \\
\text { Ghana West } \\
\text { Africa, } 1\end{array}$ & $\begin{array}{l}322 \text { children and } \\
\text { their mothers }\end{array}$ & $\begin{array}{l}\text { cross- } \\
\text { sectional }\end{array}$ & $\begin{array}{l}77 \% \text { Ghana } \\
\text { Demographic and } \\
\text { Health Survey } 2014\end{array}$ & & $\begin{array}{l}\text { Community of } \\
\text { residence of } \\
\text { mothers, lack of } \\
\text { knowledge, }\end{array}$ & \\
\hline $\begin{array}{l}\text { Adedokun1et } \\
\text { al [40], } 2017 \\
\text { Nigeria } \\
\text { West Africa, } \\
1\end{array}$ & $\begin{array}{l}5,754 \text { children aged } \\
12-23 \text { months }\end{array}$ & $\begin{array}{l}\text { Secondary } \\
\text { data } \\
\text { analysis }\end{array}$ & $\begin{array}{l}81.5 \% \text { Federal } \\
\text { Ministry of Health } \\
\text { Nigeria, } 2011\end{array}$ & Moderate & $\begin{array}{l}\text { Mothers being } \\
\text { illiterate, lack of } \\
\text { information about } \\
\text { immunization, } \\
\text { mothers not } \\
\text { attending ANC, } \\
\text { delivery at home, } \\
\text { economically dis } \\
\text { advantage } \\
\text { mothers, difficulty } \\
\text { getting to health } \\
\text { facility due to bad } \\
\text { terrines }\end{array}$ & \\
\hline
\end{tabular}




\begin{tabular}{|c|c|c|c|c|c|c|c|}
\hline $\begin{array}{l}\text { Author, year, } \\
\text { geographical } \\
\text { location and } \\
\text { number of } \\
\text { countries } \\
\text { included in } \\
\text { the study }\end{array}$ & $\begin{array}{l}\text { Participants and } \\
\text { demographic }\end{array}$ & $\begin{array}{l}\text { Study } \\
\text { design }\end{array}$ & $\begin{array}{l}\text { Reported national } \\
\text { immunization } \\
\text { coverage, Data } \\
\text { source, Date }\end{array}$ & Quality & Key childhood immu & ization reported ba & ers \\
\hline $\begin{array}{l}\text { Chidiebere et } \\
\text { al [41], } 2014 \\
\text { Nigeria } \\
\text { West Africa, } \\
1\end{array}$ & 34,596 women & $\begin{array}{l}\text { Secondary } \\
\text { data } \\
\text { analysis }\end{array}$ & Not reported & High & $\begin{array}{l}\text { Lack of } \\
\text { information about } \\
\text { immunization, fear } \\
\text { of side-effects, } \\
\text { delivery at home, } \\
\text { place of residence }\end{array}$ & $\begin{array}{l}\text { Immunization } \\
\text { centers too far }\end{array}$ & \\
\hline $\begin{array}{l}\text { Ekouevi et al } \\
\text { [42], } 2018 \\
\text { Togo } \\
\text { West Africa, } \\
1\end{array}$ & $\begin{array}{l}1,128 \text { children aged } \\
12-23 \text { months }\end{array}$ & $\begin{array}{l}\text { cross- } \\
\text { sectional }\end{array}$ & Not reported & High & $\begin{array}{l}\text { Mothers not } \\
\text { educated, low } \\
\text { income, poor road } \\
\text { conditions, lack of } \\
\text { means of } \\
\text { transportation, }\end{array}$ & $\begin{array}{l}\text { Long distance } \\
\text { to health centers }\end{array}$ & \\
\hline $\begin{array}{l}\text { Tadess et al } \\
\text { [43], } 2017 \\
\text { Ethiopia } \\
\text { East Africa, } 1\end{array}$ & $\begin{array}{l}630 \\
\text { mothers/caretakers }\end{array}$ & $\begin{array}{l}\text { Case } \\
\text { control }\end{array}$ & $\begin{array}{l}79 \% \text { Ethiopian } \\
\text { Health Sector } \\
\text { Transformation } \\
\text { Plan (HSTP), } 2009\end{array}$ & High & $\begin{array}{l}\text { Inaccessible health } \\
\text { facility, poor } \\
\text { motivation, } \\
\text { unfavorable } \\
\text { attitude and bad } \\
\text { treatment of health } \\
\text { workers, lack of } \\
\text { logistics, } \\
\text { inconvenient } \\
\text { immunization time, } \\
\text { inadequate } \\
\text { information about } \\
\text { immunization }\end{array}$ & $\begin{array}{l}\text { Vaccines } \\
\text { shortages }\end{array}$ & $\begin{array}{l}\text { Inadequate } \\
\text { communication } \\
\text { skills, lack of } \\
\text { willingness and } \\
\text { restricted } \\
\text { vaccine open } \\
\text { policy, }\end{array}$ \\
\hline $\begin{array}{l}\text { Negussie et } \\
\text { al [44], } 2016 \\
\text { Ethiopia } \\
\text { East Africa, } 1\end{array}$ & $\begin{array}{l}548 \text { children aged } \\
12 \text { to } 23 \text { months }\end{array}$ & $\begin{array}{l}\text { Case } \\
\text { control }\end{array}$ & $\begin{array}{l}\text { 24\% Ethiopia } \\
\text { Demographic and } \\
\text { Health Survey, } 2011\end{array}$ & High & $\begin{array}{l}\text { Lack of knowledge } \\
\text { about } \\
\text { immunization } \\
\text { benefits, mother's } \\
\text { negative perception } \\
\text { of vaccine side } \\
\text { effects, migration } \\
\text { of mothers }\end{array}$ & $\begin{array}{l}\text { Unavailability of } \\
\text { vaccines }\end{array}$ & \\
\hline $\begin{array}{l}\text { Bosu et al } \\
\text { [45], } 1997 \\
\text { Ghana } \\
\text { West Africa, } \\
1\end{array}$ & 469 mothers & $\begin{array}{l}\text { Cross } \\
\text { sectional }\end{array}$ & $\begin{array}{l}43 \% \text { Ministry of } \\
\text { Health Ghana } \\
\text { Maternal and Child } \\
\text { Health and Family } \\
\text { Planning. Annual } \\
\text { Report, } 1992\end{array}$ & High & $\begin{array}{l}\text { Poor knowledge } \\
\text { about } \\
\text { immunization, } \\
\text { financial } \\
\text { difficulties, long } \\
\text { waiting times, } \\
\text { transport } \\
\text { difficulties, attitude } \\
\text { of service providers } \\
\text { and fear of side- } \\
\text { effects }\end{array}$ & $\begin{array}{l}\text { Lack of suitable } \\
\text { venues and } \\
\text { furniture at } \\
\text { outreach clinics, } \\
\text { and weak inter- } \\
\text { sectoral } \\
\text { collaboration }\end{array}$ & $\begin{array}{l}\text { Poorly } \\
\text { motivated } \\
\text { service } \\
\text { providers }\end{array}$ \\
\hline $\begin{array}{l}\text { Desgrées du } \\
\text { Loû et al [46], } \\
1994 \\
\text { Senegal } \\
\text { West Africa, } \\
1\end{array}$ & $\begin{array}{l}6,078 \\
\text { Mothers } \\
\text { /caretakers }\end{array}$ & $\begin{array}{l}\text { Cross } \\
\text { sectional }\end{array}$ & $\begin{array}{l}51 \% \\
\text { WHO/EPI/CEIS/93.1 } \\
\text { (summary for the } \\
\text { WHO African } \\
\text { Region) } 1990\end{array}$ & Moderate & $\begin{array}{l}\text { Difficult } \\
\text { geographical } \\
\text { terrain (living on } \\
\text { hills), children in } \\
\text { large compound } \\
\text { with large number } \\
\text { of children. }\end{array}$ & $\begin{array}{l}\text { Distance } \\
\text { between the } \\
\text { child's village } \\
\text { and the health } \\
\text { center }\end{array}$ & \\
\hline $\begin{array}{l}\text { Sato R. } \\
\text { [47], } 2019 \\
\text { Nigeria } \\
\text { West Africa, } \\
1\end{array}$ & 28,085 children & $\begin{array}{l}\text { Secondary } \\
\text { data } \\
\text { analysis }\end{array}$ & Not reported & Moderate & $\begin{array}{l}\text { Have no faith in } \\
\text { immunization, lack } \\
\text { of awareness of } \\
\text { the need for } \\
\text { immunization, } \\
\text { poor household }\end{array}$ & $\begin{array}{l}\text { Shortage of } \\
\text { vaccine, limited } \\
\text { health centers } \\
\text { immunization } \\
\text { point is too } \\
\text { far/inconvenient }\end{array}$ & \\
\hline
\end{tabular}




\begin{tabular}{|c|c|c|c|c|c|c|c|}
\hline $\begin{array}{l}\text { Author, year, } \\
\text { geographical } \\
\text { location and } \\
\text { number of } \\
\text { countries } \\
\text { included in } \\
\text { the study }\end{array}$ & $\begin{array}{l}\text { Participants and } \\
\text { demographic }\end{array}$ & $\begin{array}{l}\text { Study } \\
\text { design }\end{array}$ & $\begin{array}{l}\text { Reported national } \\
\text { immunization } \\
\text { coverage, Data } \\
\text { source, Date }\end{array}$ & Quality & Key childhood immu & zation reported ba & iers \\
\hline $\begin{array}{l}\text { Akwataghibe, } \\
\text { N. N. et al } \\
\text { [48], 2019 } \\
\text { Nigeria } \\
\text { West Africa, } \\
1\end{array}$ & 215 children, & $\begin{array}{l}\text { Cross } \\
\text { sectional }\end{array}$ & Not reported & High & $\begin{array}{l}\text { Ethnicity, culture, } \\
\text { household decision } \\
\text { making, and gender } \\
\text { relations; } \\
\text { lack of knowledge } \\
\text { and awareness of } \\
\text { the value of } \\
\text { immunization, } \\
\text { negative beliefs } \\
\text { and attitudes } \\
\text { toward } \\
\text { immunization; past } \\
\text { experiences with } \\
\text { immunization, } \\
\text { migration }\end{array}$ & $\begin{array}{l}\text { shortage of } \\
\text { health workers, } \\
\text { unavailability of } \\
\text { vaccines at } \\
\text { scheduled } \\
\text { times; } \\
\text { Inadequate } \\
\text { electrical power } \\
\text { supply to keep } \\
\text { the vaccine cold } \\
\text { chain at } \\
\text { facilities, long } \\
\text { distance for } \\
\text { mothers in } \\
\text { "hard-to-reach" } \\
\text { areas } \\
\text { Areas }\end{array}$ & $\begin{array}{l}\text { Reminders not } \\
\text { sent on time } \\
\text { about routine } \\
\text { immunization } \\
\text { or outreach } \\
\text { days }\end{array}$ \\
\hline $\begin{array}{l}\text { Yismaw, A. E. } \\
\text { et al [49], } \\
2019 \\
\text { Ethiopia } \\
\text { East Africa, } 1\end{array}$ & $\begin{array}{l}301 \\
\text { mothers/caretakers }\end{array}$ & $\begin{array}{l}\text { cross- } \\
\text { sectional }\end{array}$ & $\begin{array}{l}86 \% \text { Federal } \\
\text { Ministry of Health } \\
(2010)\end{array}$ & High & $\begin{array}{l}\text { Lack of Knowledge } \\
\text { of next visit; and } \\
\text { lack of knowledge } \\
\text { about the benefits } \\
\text { of vaccination }\end{array}$ & $\begin{array}{l}\text { Long distance } \\
\text { to reach nearby } \\
\text { health facility }\end{array}$ & \\
\hline $\begin{array}{l}\text { Ntenda P. } \\
\text { [50], 2019 } \\
\text { Malawi } \\
\text { East Africa, } 1\end{array}$ & $\begin{array}{l}3,111 \text { children and } \\
\text { mothers }\end{array}$ & $\begin{array}{l}\text { Cross- } \\
\text { sectional }\end{array}$ & 76\% WHO ( 2015) & High & $\begin{array}{l}\text { Children born to } \\
\text { mothers without } \\
\text { education, children } \\
\text { poor households, } \\
\text { mothers with many } \\
\text { sibling, children } \\
\text { whose delivery } \\
\text { occurred at home, }\end{array}$ & $\begin{array}{l}\text { Inadequate } \\
\text { health facility } \\
\text { for vaccination, } \\
\text { long distance to } \\
\text { the nearest } \\
\text { health facility }\end{array}$ & \\
\hline $\begin{array}{l}\text { Okenwa, U. J. } \\
\text { et al [51], } \\
2019 \text { Nigeria } \\
\text { West Africa, } \\
1\end{array}$ & $\begin{array}{l}344 \text { mothers } \\
\text { and their infant }\end{array}$ & $\begin{array}{l}\text { Cross- } \\
\text { sectional }\end{array}$ & Not reported & High & $\begin{array}{l}\text { Lack of awareness } \\
\text { on timing of valid } \\
\text { vaccine, }\end{array}$ & $\begin{array}{l}\text { Vaccine stock- } \\
\text { out at the } \\
\text { immunization } \\
\text { site }\end{array}$ & \\
\hline $\begin{array}{l}\text { Mthiyane, T. } \\
\text { N et al [52], } \\
2019 \\
\text { South Africa } \\
\text { South Africa } \\
1\end{array}$ & $\begin{array}{l}847 \text { eligible } \\
\text { children aged 12- } \\
59 \text { months }\end{array}$ & $\begin{array}{l}\text { Secondary } \\
\text { data } \\
\text { analysis }\end{array}$ & $\begin{array}{l}\text { 66\% WHO/UNICEF } \\
(2015)\end{array}$ & High & $\begin{array}{l}\text { Low household } \\
\text { monthly income, } \\
\text { unfriendly health } \\
\text { workers, transport } \\
\text { costs to reach the } \\
\text { clinic for } \\
\text { immunization } \\
\text { services }\end{array}$ & $\begin{array}{l}\text { Vaccine } \\
\text { shortages; } \\
\text { long distances } \\
\text { to travel to the } \\
\text { clinic }\end{array}$ & $\begin{array}{l}\text { Low level of } \\
\text { education of } \\
\text { the primary } \\
\text { caregiver, }\end{array}$ \\
\hline $\begin{array}{l}\text { Mekonnen, A. } \\
\text { G. et al [53], } \\
2019 \\
\text { Ethiopia } \\
\text { East Africa, } 1\end{array}$ & $\begin{array}{l}566 \text { children aged } \\
12-23 \text { months and } \\
\text { their } \\
\text { mothers/caregivers }\end{array}$ & $\begin{array}{l}\text { Cross } \\
\text { sectional }\end{array}$ & $\begin{array}{l}39 \% \text { Ethiopian } \\
\text { demographic } \\
\text { health survey report } \\
\text { (2016) }\end{array}$ & High & $\begin{array}{l}\text { Forgotten } \\
\text { appointment date, } \\
\text { the experience of } \\
\text { child sickness with } \\
\text { previous } \\
\text { vaccination, and } \\
\text { disrespectful } \\
\text { behavior of health } \\
\text { professionals }\end{array}$ & $\begin{array}{l}\text { Long distances } \\
\text { to travel to the } \\
\text { clinic }\end{array}$ & \\
\hline $\begin{array}{l}\text { Ibraheem, R. } \\
\text { et al [54], } \\
2019 \\
\text { Nigeria } \\
\text { West Africa, } \\
1\end{array}$ & 480 mother-infant & $\begin{array}{l}\text { cross- } \\
\text { sectional }\end{array}$ & $\begin{array}{l}\text { 53\% Nigeria } \\
\text { immunization } \\
\text { coverage survey } \\
(2016)\end{array}$ & High & $\begin{array}{l}\text { Lack of antenatal } \\
\text { care visit, } \\
\text { vaccination on } \\
\text { weekend/public } \\
\text { holidays } \\
\text { lower educational } \\
\text { level }\end{array}$ & & \\
\hline
\end{tabular}

\section{Parental/caretaker Barriers}

In this systematic review, several cited parental/caretakers' barriers were modifiable (knowledge, misconception, trust, delivery at home, long waiting time, providers' hostility, parent's forgetfulness, inconvenient time and language barrier). It was revealed that parent perception influence immunization of their children $[1,12,13,14,21,34-44,48,49]$. Parents not being knowledgeable of immunization was the most frequently and consistently reported barrier to 
childhood immunization $[2,7,11,13,15-18,22,24-25,27-28,30,34-36,3-40,42,44-45,47-50,52]$. Wiysonge et al, 2012 stated that "low parental knowledge of immunization and/or lack of access to information about childhood immunization could be an important contributor to the high burden of unimmunized children in sub-Saharan Africa". Four studies $[3,13,25,26]$, noted that a child born to a mother with little or no knowledge of vaccination may not complete the required vaccine series. Two articles reported that delay on vaccine birth doses is associated to maternal education [18, 20]. Misconceptions about childhood immunization were recorded as major hindrance to effective utilization of immunization services in this review [1, 2, 11$14,21-22,29,31,32,48,49]$ One article [17] reported that some parents believed that the immunity induced by vaccines is less effective than that of the natural disease, and they prefer to endure the diseases than immunization. Some caregivers were reported to belief in the efficacy of traditional medicines as an alternative to immunization and concomitant treatment by traditional healers [31, 35]. Lack of trust towards vaccines was a major reported barrier. Some community members were reported to refuse immunization services due to the belief that vaccines were harmful, 'expired' and could cause 'physical disability and/or death' among their children $[2,17,21-22,30,32-33,35,38,45,48,53]$.

Another important barrier noted was the role of male partners in the decision for childhood vaccination. They were often cited as being against vaccinating the children. The place of delivery of a baby was reported as determinants of full immunization of a child. Delivery at health facility enhances full immunization $[18,20,22,27,28,40,48]$. Long waiting time at health facilities was frequently and consistency noted [11, 12, 31, 35, 36, 45]. Providers' hostility and rude attitudes to mothers were also a reported immunization barrier in this review $[15,16,24,30-33,36,45,52,53]$. Two article [35, 53], noted that parents sometimes forgot the appointment date for the next immunization visit of their children. Others reported place/time for vaccination being unknown [11]. Inconvenient immunization time such as on weekend/public holidays was reported as a barrier [43, 54]. One study [3], indicated language as a barrier to childhood immunization.

On the other hand, we also recorded non-modifiable childhood immunization barriers of parents/caretakers. Those categorized as unmodifiable are factors that are extrinsic to the parent / provider dyad. These include occupation, financial limitations, place of residence of mother/caretaker, religion, ethnicity, family size, male partners' support, and migration; seasonal farm work, feeling ashamed of poverty-associated reasons and being a single mother. The decision for immunization was generally a joint decision between the mother and father of the child. But it was noted with strong emphasis that women were in charge of taking children for immunization and sometimes the husbands opposed immunization and stopped their wives from immunizing their children by denying them the social and financial support necessary $[3,16,17,22,33,38]$. The nature of occupation of the mother/caretaker was reported as a major determinant to childhood immunization [1, 11, 14, 15, 21-22, 25, 30]. Housewives were reported to have complied with higher coverage of full immunization status than other occupations such as merchants or public/private employees [11]. Also, mothers/caretakers were reported to be affected by seasonal factors. One study [1] stated that, "usually in the first quarter of the year in which most mothers engaged in coffee collection and processing in coffee processing industries often did not bring their children to the next immunization schedule". Financial limitation was a major barrier cited to hinder childhood immunization. With no money for transportation, the only way to reach immunization point would be walking, often over long distances [7, 11, 13, 15, 17, 24, 27, 34, 36, 38, 40, 42, 45, 47, 50, 52]. The place of residence of the mother was reported as determinants of full immunization of a child [39, 41,37]. One study noted that the likelihood of vaccination of a child by day 7 is higher among children residing in rural areas than those in urban and pre-urban settings [18]. Socio-cultural factors and religion were noted to have negatively impacted immunization uptake [12, 18, 19, 22, 23, 37, 48]. Ethnicity and cultural beliefs were reported barriers to vaccine utilization and coverage; certain ethnic groups within the same country were identified with low coverages. Family size was associated with the probability of a child being fully immunized. It was revealed that children from large families have low vaccine uptakes, considering the burden of other children at home in taking up immunization services $[11,13,25,46,50]$. Migration was also cited as a hindrance to childhood immunization coverage [30, 35, 44, 48]. Feeling ashamed of poverty-associated reasons was reported as barrier. Schwarz et al. 2009 indicated that, "mothers who felt that they could not dress smartly enough for the approval of other women at the clinic were less likely to attend" [24]. Babirye et al. 2011, further revealed that "poor mothers often felt stigmatized and bullied from other women and health workers if they did not show up in good clothing" [17] Being a single mother was also a cited barrier to childhood immunization in this review [37].

\section{Health System Barriers}

We noted health system barriers in this review. These includes broken cold chain, irregular supplies and distribution of vaccines; limited human resource and infrastructures, and long distances separating health facilities from families [1-3, 11, 13, 14-16, 19, 22, 23, 25, 29, 32, 35, 43, 44, 47-52]. Vaccine shortages at health facility level and difficulties of transporting vaccines were commonly reported to significantly hinder immunization service [1-174 3 , $11,14,16,19,22,23,25,33,43,47,48,52,51]$. Some facilities were reported to have utilized vaccine refrigerators from nearby health centers due to poor working condition of theirs [1,3,32]. It was noted that due to staff limitation, often only one staff conducted immunization sessions in the catchment population $[2,3,11,25]$. Studies $[11,13,45,47-50,52,53]$ also revealed that some hard-to-reach areas do not have health facilities nearby to provide childhood immunization.

It was reported that, caretakers covered long distances to reach immunization centers resulted to non-completion of vaccination series [2, 3, 13, 18, 21$26,31,36,38,40-43,46]$. Some studies $[11,13,18,26]$ attempted to study the associations of distance with immunization outcomes. Tefera et al, 2018. indicated that "families whose home was at least an hour from the vaccination site were less likely to be fully vaccinated (56\%) than families whose home was between 30 and 59 min away (67\%)". According to Miyahara et al. 2016, "the longer the distance from vaccination site, the lower the chances of vaccination by day 7 (of life) of a child". Poor arrangement and coordination of immunization seasons at health center level were identified as barrier $[16,38,43,45]$. 


\section{Providers Barriers}

In addition to the parental and health system barriers mentioned above, providers were identified as possessing barriers to immunization. These factors include the lack of knowledge of vaccine indications and contraindications and the lack of counseling skills [3, 16, 43, 52]. Health workers were reported to cover long distances on outreach services due to inadequate health centers $[11,13,15,43]$. The restricted vaccine opening policy (Use of multi-dose vails and the limited time for their use) was noted as a barrier specifically for the BCG vaccine [18, 30, 43]. It was also cited that reminders were not sent on time about routine immunization or outreach days [48]

\section{Discussion}

Our review aims at identifying major childhood immunization barriers confronting health systems, providers, and parents across Sub-Saharan Africa. Understanding of these barriers will help inform decision-makers and other relevant players involved in immunization programs, and to guide health interventions aims at improve immunization coverage. The study revealed childhood immunization barriers affecting utilization and coverage in the region. We grouped these barriers under three separate domains: barriers inherent in the parents/caretakers, those specific to the health system, and those related to the providers. Parental barriers were the most commonly and consistently identified than providers and health systems. Several of the cited parental/caretakers' barriers were unmodifiable. Parents/caretakers reported barriers include lack of knowledge, misconceptions, hostile attitude of health providers, distance, financial deprivation, lack of partners' support, and distrust of the medical systems. Other associated factors include the number of offspring, life style, migration, place of residence, long waiting time, parent's forgetfulness; inconvenient time, being a single mother, occupation, language barrier, seasonal farm work, and feeling ashamed of poverty-associated reasons. Health system barriers include inadequate infrastructures and cold chain maintenance; and poor coordination. Providers' constraints include limited human resources and knowledge.

Knowledge of vaccines is very important for effective vaccine acceptance and utilization by parents. Low vaccination coverage in children is largely a result on the lack of knowledge of vaccines of healthcare providers and parents. Parents with low education and low socioeconomic status attainment showed more uncertainty towards immunization $[3,11,1315-18,22,24-25,27-28,30,34-36,3-40,42,44-45,48,49]$. This result was also mirrored in another systematic review conducted in middle and low income countries which revealed that, low educational level and low socioeconomic status were often strongly correlated, and were linked to low vaccine uptake; however, the underlying explanations for these associations were rarely investigated [55]. Thus health education programs targeting these groups are critical in increasing vaccines acceptance, utilization and coverage. Healthcare providers should receive training in medical ethics to promote the culture of treating parents/caretakers with dignity and respect. We noted that parents held reservations towards the associated side effects of vaccines. Other expressed a total distrust of immunization programs and vaccines [2, 17, 21-22, 30, $3233,35,38,45,47,48]$. This is in line with previous review of Influenza Vaccine hesitancy, which pointed out that, a lack of confidence due to low perceived effectiveness of the vaccine was a hindrance to vaccine uptake [56]. Another review outlined similar beliefs, including concerns about side effects, skepticism toward vaccine safety, and belief in conspiracy theories [59]. To overcome this, immunization programs should intensify public sensitization on vaccines safety and promote effective mechanisms of addressing parents' concerns. Healthcare workers should develop approaches that acknowledge parental concerns and respectfully try to correct their misconceptions. The attitude of male partners against immunization is often noted in this review. A study carried out in Cambodia suggested that women's decision making power and autonomy were relevant to maternal and child health outcomes [60]. It is important to carefully consider the social contexts during program design and implementation for child immunization. We need to effectively address socio-cultural contexts by involving the entire community, and not only target mothers and female caregivers. The review also raised the pressing need for women to be empowered to overcome their financial challenges in taking their children to vaccination centers.

Equally challenging is overcoming health system barriers identified, including staff shortage, the cost of maintaining the cold chain, storage and transportation of vaccines and consumables. The long distances between health centers and the families they serve are barrier that require systemic policy changes to address. The data suggest that countries should increase government financial gross domestic product (GDP) allocation to their health sector, consistent with the recommendation in the Abuja declaration [49]. Increased financial resources would enable countries to equip and upgrade existing health facilities and to increase their numbers. Targeted resources may motivate and enable staff deployed in remote areas for effective outreach activities to maximize coverage of immunization. Poor arrangement and coordination of immunization seasons at health center level was noted [16, 38, $43,45]$ a review (conducted in sub-Saharan countries) focus on children and youth noted that, chaotic and uncoordinated services can cause delays and increase costs for beneficiaries [62]. A coordinated National Immunization Program can rationalize services, thus improve immunization uptake and regulating healthcare providers.

In this review of barriers to childhood immunization, the parental/caretakers' barriers are the most prominent, followed by health systems and providers' barriers. It corroborate with a systematic review (studies undertaken across countries) which noted that, most factors were linked with family features and parents' knowledge and attitudes; this is complex and more difficult to address, requiring targeted intervention [63]. A published article exploring vaccine hesitancy stated that different attitudes tend to group into definite profiles; for example, anti-vaccination attitudes could be attributed to ignorance, misinformation, irrationality or it could be positively correlated with vaccine-related knowledge [64]. This finding disagreed with previous systematic review conducted in middle and low income countries. It noted that majority of the reasons linked to being under-vaccinated were related to health care system, and fewer due to parental attitudes and knowledge [55]. Some of the barriers cited may be modifiable within the constraints of overstretched health systems. Others may require systemic policy changes to address. Some factors related to health care system may be feasible to develop approaches that can be adapted to and implemented in a range of settings, such as training of health workers to reduce missed opportunities, improve communication, and remove barriers by enhancing outreach services

Page $11 / 16$ 


\section{Study Limitations}

Our study has few limitations. As most literatures cited are observational in nature, this study cannot confirm causation nor completely rule out confounding. A few studies also relied on survey data $[7,12,18,27,37,40,41]$ with the potential for selection or nonresponse bias. Population-base data studies may be liable to misclassification or measurement error, leading to information biases. Retrospective studies of caretakers/parents beliefs are subject to recall bias. Majority of the studies were conducted in East Africa [1, 3, 11-13, 16, 17, 19, 20, 21, 25, 27-28, 32, 34, 38, 43-44] and West Africa $[1,15,18,22-24,29-31,33,36-37,39-42,45-46]$ limiting generalizability to the rest of the continent. A quantitative meta-analysis from these studies may have been useful for analyzing quantitative trends, although the heterogeneity of the studies precluded such analyses.

\section{Conclusion}

Although various methods of improving vaccination coverage in Sub-Saharan Africa have been identified, achieving the desired levels for the realization of the fullest benefits of immunization is still a major challenge. This can be achieved through combined efforts of healthcare systems and providers; and address people, the communities and societies in which they live. Aggregation of known immunization barriers and the evidence on effective interventions to address these barriers should be core component of immunization programs in Sub-Saharan Africa and elsewhere.

\section{Abbreviations}

DTP3 Diphtheria

Tetanus-Pertussis Vaccine third dose

BCG Bacillus Calmette

Guérin

\section{Declarations}

\section{Author's contributions}

JBB and SX conceived the review protocol. JBB, DQ, FO were involved in the data extraction and quality assessment. JBB, LC, SX involved in data synthesis and creation of table; JBB drafted the manuscript with input from all authors. All authors read and approved the final manuscript and submission.

\section{Ethics approval and consent to participate}

Not applicable

\section{Consent for publication}

Not applicable

\section{Availability of data and material}

Not applicable

Competing interests

We declare none

\section{Funding}

China Medical Board funded the study. Grant number [CMB-14-188]; the funding agency did not play any role in the preparation of this study; study design, data collection, analysis, writing and decision to submission.

\section{Authors' contributions}

JBB and SX conceived the review protocol. JBB, DQ, FO were involved in the data extraction and quality assessment. JBB, LC, SX involved in data synthesis and creation of table; JBB drafted the manuscript with input from all authors. All authors read and approved the final manuscript and submission.

\section{Acknowledgements}

We would like to thank Emmanuel Osman Kamara from the Language Art Department, Earnest Bai Koroma University of Science and Technology, Sierra Leone for his assistance with English Language editing. 


\section{References}

1. Tadesse H, Deribew A, Woldie M. Explorative assessment of factors affecting child immunization in Wonago district, Gedeo zone, South Ethiopia. Arch Med Sci 2009; 5, 2: 233-240

2. Obasoha PE, Mustapha MA, Makada A, Obasohan DN. Evaluating the Reasons for Partial and Non-immunization of Children in Wushishi Local Government Area, Niger State, Nigeria: Methodological Comparison. African Journal of Reproductive Health 2018; 22:113:

https://doi.org/10.29063/ajrh2018/v22i4.12

3. Malande OO, Munube D, Afaayo RN, Annet K, Bodo B, Bakainaga A, Ayebare E, Njunwamukama S, Mworozi A E, Musyoki MA. Barriers to effective uptake and provision of immunization in a rural district in Uganda. PloS one 2019; 14:2 e0212270, https://doi.org/10.1371/journal.pone.0212270

4. Wolfson LJ, Gasse F, Lee-Martin S-P, Lydon P, Magan A, Tibouti A, et al. Estimating the costs of achieving the WHO-UNICEF Global Immunization Vision and Strategy, 2006-2015. Bulletin of the World Health organization 2008; 86:27. https://doi.org/10.2471/blt.07.045096

5. World health Organization. World health statistics 2010: World Health Organization; 2010. Available from: https://www.who.int/whosis/whostat/2010 Accessed: April 9,2019

6. GBD 2016 Causes of Death Collaborators (2017). Global, regional, and national age-sex specific mortality for 264 causes of death, $1980-2016$ : a systematic analysis for the Global Burden of Disease Study 2016. Lancet (London, England), 390(10100), 1151-1210. https://dx.doi.org/10.1016\%2FS0140-6736(17)32152-9

7. Wiysonge CS, Uthman OA, Ndumbe PM, Hussey GD. Individual and contextual factors associated with low childhood immunisation coverage in subSaharan Africa: a multilevel PloS one 2012; vol. 7, 5 e37905. https://doi.org/10.1371/journal.pone.0037905

8. Global Vaccine Action Plan - Secretariat Annual Report 2016 Available from: who.int/immunization/global_vaccine_action_plan/gvap_secretariat_report_2016 Accessed: April 4,2019

9. WHO - Ministerial Conference on Immunization in Africa. Available from: https://www.who.int/immunization/1st...ministerial_conference/en/ Accessed: 6 April, 2019

10. Cooper S, Betsch C, Sambala EZ, Mchiza N, Wiysonge CS: Vaccine hesitancy - a potential threat to the achievements of vaccination programmes in Africa. Hum Vaccin Immunother. 2018; 14: 2355. https://doi.org/10.1080/21645515.2018.1460987

11. Tefera AY, Wagner AL, Mekonen BE, Carlson FB, Boulton LM. Predictors and Barriers to Full Vaccination among Children in Ethiopia. Vaccines 2018; vol. 6:22 https://doi.org/10.3390/vaccines6020022

12. Porth JM, WagnerAL, Teklie H, Abeje Y, Moges B, Boulton ML. Vaccine non-receipt and refusal in Ethiopia. The expanded program on immunization coverage survey, 2012. Vaccine 2019; 37:2106 https://doi.org/10.1016/j.vaccine.2019.02.045

13. Kiptoo E, Kobia G, Moses M, Ngure R. Factors Influencing Low Immunization Coverage Among Children Between 12 - 23 Months in East Pokot, Baringo County, Kenya. J Vaccines 2015; 1: 00012 https://10.15406/ijvv.2015.01.00012

14. Cockcroft A, Usman MU, Nyamucherera OF, Emori H, Duke B, Umar NI, Andersson N Why children are not vaccinated against measles a crosssectional study in two Nigerian Archives of public health 2014; 72:48 http://www.archpublichealth.com/content/72/1/48

15. Nolna SK, Bonono CR, Moncher MN, Bind T, Nolna D, Zogo PO. Factors influencing the performance of routine immunization in urban areas: A comparative case study of two cities in Cameroon Douala and Yaoundé. Vaccine 2018; 36:7549 https://doi.org/10.1016/j.vaccine.2018.10.048

16. Zewdie A, Letebo M, Mekonnen T. Reasons for defaulting from childhood immunization program: a qualitative study from Hadiya zone, Southern Ethiopia. BMC Public Health 2016; 16:1240 https://doi.org/10.1186/s12889-016-3904-1

17. Babirye J N, Rutebemberwa E, Kiguli J, Wamani H, Nuwaha F, Engebretsen IMS. More support for mothers: a qualitative study on factors affecting immunisation behaviour in Kampala Uganda. BMC public health 2011;11:723. https://doi.org/10.1186/1471-2458-11-723

18. Miyahara R, Jasseh M, Gomez P, Shimakawa Y, Greenwood B, Keita K, Ceesay S, D’Alessandro U, Roca A. Barriers to timely administration of birth dose vaccines in The Gambia, West Africa. Vaccine 2016; vol.34 29:3335 https://doi.org/10.1016/j.vaccine.2016.05.017

19. Pertet AM, Kaseje D, Otieno-Odawa CF, Kirika L, Wanjala C, Ochieng J, Jaoko M, Otieno W, Odindo D. Under vaccination of children among Maasai nomadic pastoralists in Kenya: is the issue geographic mobility, social demographics or missed opportunities. BMC Public Health 2018; 18:1389 https://doi.org/10.1186/s12889-018-6309-5

20. Yenit MK, Gelaw YA, Shiferaw AM. Mothers' health service utilization and attitude were the main predictors of incomplete childhood vaccination in east-central Ethiopia: a case-control study. Archives of Public Health 2018; 76:14 https://doi.org/10.1186/s13690-018-0261-9

21. Tugumisirize F, Tumwine JK, Mworozi EA. Missed opportunities and caretaker constraints to childhood vaccination in rural area in Uganda. East Africa medical journal. 2002; 79: 7

22. Babalola S. Maternal reasons for non-immunisation and partial immunisation in northern Nigeria. Journal of Paediatrics and Child Health 2011 ; 47:276 https://doi.org/10.1111/j.1440-1754.2010.01956.x

23. Oladokun RE, Adedokun BO, Lawoyin TO. Children not receiving adequate immunization in Ibadan, Nigeria: What reasons and beliefs do their mothers have? Nigerian Journal of Clinical Practice 2010; Vol. 13(2):173-178

24. Schwarz NG, Gysels M, Pell C, Gabor J, Schlie M, Issifou S, Lell B, Kremsner PG, Grobusch MP, Pool R. Reasons for non-adherence to vaccination at mother and child care clinics (MCCs) in Lambaréné, Gabon. Vaccine 2009; 27:5371-5375 https://doi.org/10.1016/j.vaccine.2009.06.100

25. Ismail IT, El-Tayeb EM, Omer MD, Eltahir YM, El-Sayed ET, Deribe K. Assessment of Routine Immunization Coverage in Nyala Locality, Reasons behind Incomplete Immunization in South Darfur State, Sudan. Asian journal of medical sciences 2014; 25;6(1):1-8. https:/doi.org/10.19026/ajms.6.5348

Page 13/16 
26. Rees H, Buch E, Ferrinho P. De L.G.M, Groenewald HT, Neethling A. Immunisation coverage and reasons associated with non-immunisation in Alexandra Township, September 1988. South Africa Medical Journal 1991: Vol. 80(8) 378-381

27. Nadella P, Smith ER. Muhihi, A, Noor RA, Masanja H, Fawzi, WW, Sudfeld, CR. Determinants of delayed or incomplete diphtheria-tetanus-pertussis vaccination in parallel urban and rural birth cohorts of 30,956 infants in Tanzania. BMC Infectious Diseases 2019; 19 : 188 https://doi.org/10.1186/s12879-019-3828-3

28. Meleko A, Geremew M, Birhanu F. Assessment of Child Immunization Coverage and Associated Factors with Full Vaccination among Children Aged 12-23 Months at Mizan Aman Town, Bench Maji Zone, Southwest Ethiopia. International Journal of Pediatrics 2017; 7976587 https://doi.org/10.1155/2017/7976587

29. Itimi K, Dienye PO, Ordinioha B. Community participation and childhood immunization coverage: A comparative study of rural and urban communities of Bayelsa State, south-south Nigeria. Niger Med J. 2012; 53:21-25. https://dx.doi.org/10.4103\%2F0300-1652.99826

30. Kagoné M, Yé M, Nébié E, Sié A, Müller O, Beiersmann C. Community perception regarding childhood vaccinations and its implications for effectiveness: a qualitative study in rural Burkina Faso. BMC Public Health 2018; 18:324 https://doi.org/10.1186/s12889-018-5244-9

31. Tobin-West $\mathrm{Cl}$, Alex-Hart BA. Identifying barriers and sustainable solution to childhood immunization in Khana local government area of Rivers State, Nigeria. Int Q Community Health Educ. 2011-2012; 32:149-58. http://dx.doi.org/10.2190/IQ.32.2.e

32. Braka F, Asiimwe D, Soud F, Lewis RF, Makumbi I, Gust D. A qualitative analysis of vaccine safety perceptions and concerns among caretakers in Uganda. Matern Child Health J. 2012; 16:1045-52. https://doi.org/10.1007/s10995-011-0826-5

33. Ambe JP, Omotara BA, Mandu Baba M. Perceptions, beliefs and practices of mothers in sub-urban and rural areas towards measles and measles vaccination in Northern Nigeria. Tropical Doctors. 2001; 31:89-90. https://doi.org/10.1177\%2F004947550103100211

34. Tadesse H, Deribew A, Woldie M. Predictors of defaulting from completion of child immunization in south Ethiopia, May 2008: a case control study. BMC Public Health. 2009; 9:150. https://dx.doi.org/10.1186\%2F1471-2458-9-150

35. Jani JV, De Schacht C, Jani IV, Bjune G. Risk factors for incomplete vaccination and missed opportunity for immunization in rural Mozambique. BMC Public Health. 2008; 8:161. https://dx.doi.org/10.1186\%2F1471-2458-8-161

36. Eng E, Naimoli J, Naimoli G, Parker KA, Lowenthal N. The Acceptability of Childhood Immunization to Togolese Mothers: A Sociobehavioral Perspective. Health Educ Q. 1991; 18:97-110.

37. Landoh DE, Ouro-Kavalah F, Yaya I, Kahn AL, Wasswa P, Lacle A, Nassoury DI, Gitta SN, Soura AB. Predictors of incomplete immunization coverage among one to five years old children in Togo. BMC Public Health. 2016; 16:968. https://dx.doi.org/10.1186\%2Fs12889-016-3625-5

38. Legesse E, Dechasa W. An assessment of child immunization coverage and its determinants in Sinana District, Southeast Ethiopia. BMC Pediatr. 2015; 15:31. https://dx.doi.org/10.1186\%2Fs12887-015-0345-4

39. Wemakor A, Helegbe GK, Abdul-Mumin A, Amedoe S, Zoku JA, Dufie Al. Prevalence and factors associated with incomplete immunization of children (12-23 months) in Kwabre East District, Ashanti Region, Ghana. Arch Public Health. 2018; 76:67 https://dx.doi.org/10.1186\%2Fs13690-018-0315-z

40. Adedokun ST, Uthman OA, Adekanmbi VT, Wiysonge CS. Incomplete childhood immunization in Nigeria: a multilevel analysis of individual and contextual factors. BMC Public Health. 2017; 17:236 https://dx.doi.org/10.1186\%2Fs12889-017-4137-7

41. Chidiebere ODI, Uchenna E, Kenechi OS. Maternal sociodemographic factors that influence full child immunisation uptake in Nigeria. South African Joumal of Child Health, 2014; v. 8, n. 4, p. 138-142, DOI:10.7196/SAJCH.661

42. Ekouevi DK, Gbeasor-Komlanvi FA, Yaya I, Zida-Compaore WI, Boko A, Sewu E, Lacle A, Ndibu N, Toke Y, Landoh DE. Incomplete immunization among children aged 12-23 months in Togo: a multilevel analysis of individual and contextual factors. BMC Public Health. 2018; 18:952. doi: https://dx.doi.org/10.1186\%2Fs12889-018-5881-z

43. Tadesse T, Getachew K, Assefa T, Ababu Y, Simireta T, Birhanu Z, Hailemichael Y. Factors and misperceptions of routine childhood immunization service uptake in Ethiopia: findings from a nationwide qualitative study. Pan Afr Med J. 2017; 5:28 https://dx.doi.org/10.11604\%2Fpamj.2017.28.290.14133

44. Negussie A, Kassahun W, Assegid S, Hagan AK. Factors associated with incomplete childhood immunization in Arbegona district, southern Ethiopia: a case--control study. BMC Public Health. 2016; 16:27. https://dx.doi.org/10.1186\%2Fs12889-015-2678-1

45. Bosu WK, Ahelegbe D, Edum-Fotwe, E, Bainson, KA, Turkson, P. K. Acta Tropica 1997; 68:259-267 https://doi.org/10.1016/S0001-706X(97)00094-6

46. Desgrées du Loû A, Pison G. Barriers to universal child immunization in rural Senegal 5 years after the accelerated Expanded Programme on Immunization. Bull World Health Organ. 1994; 72:751-759.

47. Sato R. Differential determinants and reasons for the non- and partial vaccination of children among Nigerian caregivers. 2019; 38(1), 63-69. doi:10.1016/j.vaccine.2019.09.097

48. Akwataghibe NN, Ogunsola EA, Broerse J, Popoola OA, Agbo Al, Dieleman MA. Exploring Factors Influencing Immunization Utilization in Nigeria-A Mixed Methods Study. Frontiers in public health. 2019; 7, 392. doi:10.3389/fpubh.2019.00392

49. Yismaw,A E, Assimamaw, NT, Bayu, N, Mekonen SS. Incomplete childhood vaccination and associated factors among children aged $12-23$ months in Gondar city administration, Northwest, Ethiopia 2018. BMC research notes. 2019 12(1), 241. doi:10.1186/s13104-019-4276-2

50. Ntenda P. Factors associated with non- and under-vaccination among children aged 12-23 months in Malawi. A multinomial analysis of the population-based sample. Pediatrics and neonatology. 2019; 60(6), 623-633. doi:10.1016/j.pedneo.2019.03.005 
51. Okenwa UJ, Dairo MD, Uba B, \& Ajumobi O. Maternal reasons for non-receipt of valid Hepatitis B birth dose among mother-infant pairs attending routine immunization clinics, South-east, Nigeria. 2019; 37(46), 6894-6899. doi:10.1016/j.vaccine.2019.09.056

52. Mthiyane TN, Cohen C, Norris S A, Walaza S, Tempia S, Cohen AL, Von Mollendorf, C. Factors associated with missed and delayed DTP3 vaccination in children aged 12 - 59 months in two communities in South Africa, 2012 - 2013. South African medical journal. 2019; 109(8), 562-569. doi:10.7196/SAMJ.2019.v109i8.13244

53. Mekonnen AG, Bayleyegn, AD, Ayele ET. Immunization coverage of 12-23 months old children and its associated factors in Minjar-Shenkora district, Ethiopia: a community-based study. BMC pediatric. 2019; 19(1), 198. doi:10.1186/s12887-019-1575-7

54. Ibraheem R, Abdulkadir M, Akintola M, Adeboye, M. Determinants of Timely Presentation for Birth Dose Vaccination at an Immunization Centre in North-central Nigeria. Annals of global health. 2019; 85(1), 20. doi:10.5334/aogh.725

55. Rainey JJ, Watkins M, Ryman TK, Sandhu P, Bo A, Banerjee K. Reasons related to non-vaccination and under-vaccination of children in low and middle income countries: findings from a systematic review of the published literature,1999-2009. Vaccine. 2011; 29(46):8215-21.

56. Schmid P, Rauber D, Betsch C, Lidolt G, Denker ML. Barriers of Influenza Vaccination Intention and Behavior - A Systematic Review of Influenza Vaccine Hesitancy, 2005 - 2016. PloS one. 2017; 12(1), e0170550. https://doi.org/10.1371/journal.pone.0170550

57. Moher D, Liberati A, Tetzlaff J, Altman DG, PRISMA Group. Preferred Reporting Items for Systematic Reviews and Meta Analyses: The PRISMA Statement. PLoS Med 6(7): e1000097. https://doi.org/10.1371/journal.pmed.1000097

58. Pereira AJ, Quach S, Heidebrecht LC, Quan DS, Kolbe F, Finkelstein M, Kwong JC. Barriers to the use of reminder/recall interventions for immunizations: A systematic review. BMC Medical Informatics and Decision Making. 2012; 12:145 http://www.biomedcentral.com/1472$6947 / 12 / 145$

59. Wilson L, Rubens-Augustson T, Murphy M, Jardine C, Crowcroft N, Hui C, Wilson K. Barriers to immunization to newcomers: A systematic review. 2018; 36(8):1055-1062. doi: 10.1016/j.vaccine.2018.01.025

60. Forder JA. Attitudes Towards Immunization in Cambodia: A Qualitative Study of Health Worker and Community Knowledge, Attitudes and Practices in Kompong Chhnang 2002.

61. WHO | The Abuja Declaration: Available from: https://www.who.int/healthsystems/publications/abuja_declaration Date accessed 10 April, 2019

62. Sullivan BJ, Esmaili BE, Cunningham CK. Barriers to initiating tuberculosis treatment in sub-Saharan Africa: a systematic review focused on children and youth. Global health action. 2017; 10(1), 1290317. https://doi.org/10.1080/16549716.2017.1290317

63. Tauil MC, Sato AP, Waldman EA. Factors associated with incomplete or delayed vaccination across countries: A systematic review. Vaccine. 2016; 34 , 2635-2643 http://dx.doi.org/10.1016/j.vaccine.2016.04.016

64. Peretti-Watel P, Larson HJ, Ward JK, Schulz WS, Verger P. Vaccine hesitancy: clarifying a theoretical framework for an ambiguous notion. PLoS Curr Outbreaks. 2015; http://dx.doi.org/10.1371/currents.outbreaks.6844c80ff9f5b273f34c91f71b7fc289

\section{Additional File (Supplementary Materials)}

- File format.pdf

- Title of data:

1. Database search terms and history

2. Methodological Quality Assessment tool for Qualitative and Quantitative studies

3. Full-text articles excluded with reasons

\section{Figures}




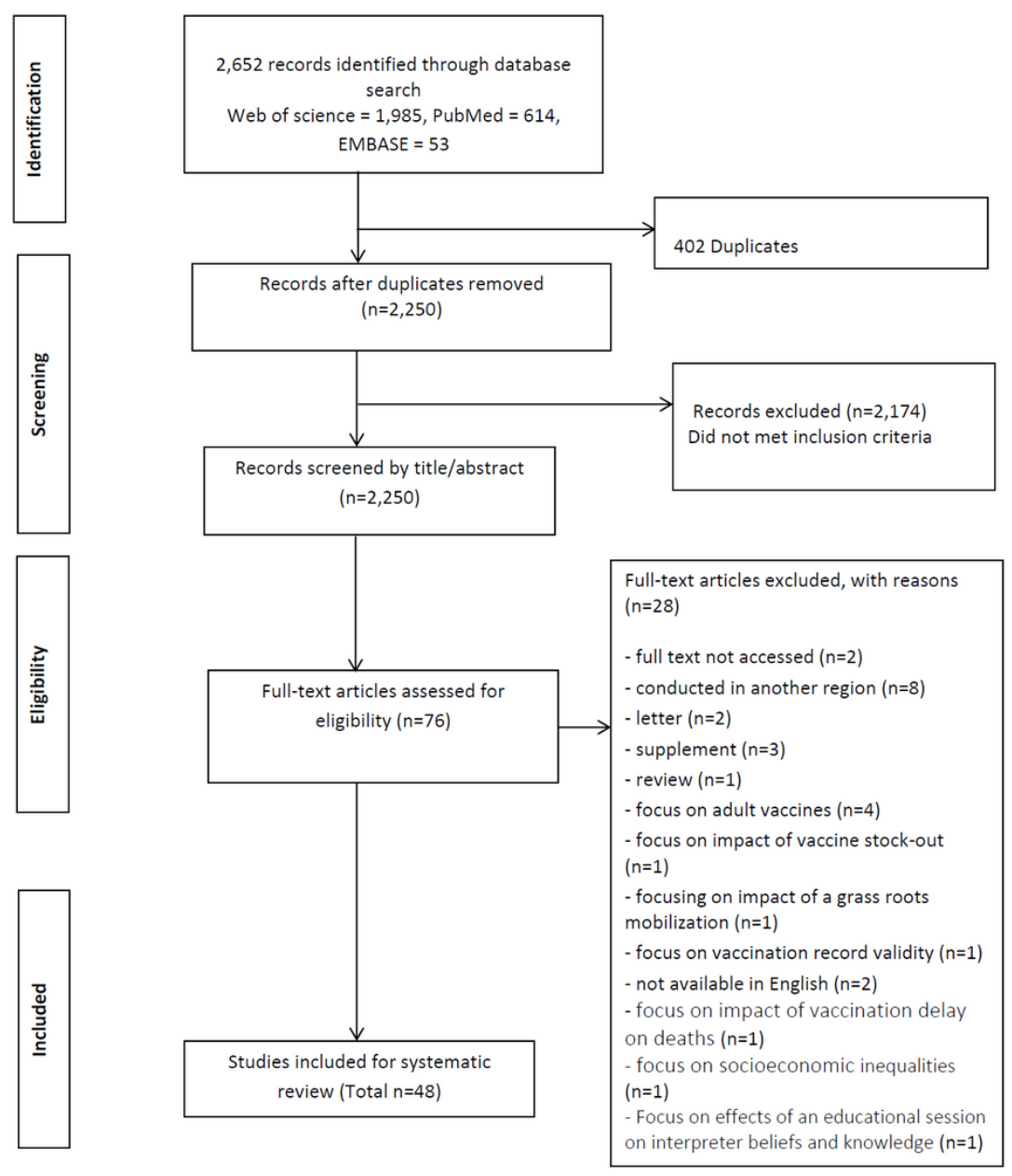

Figure 1

Study selection

\section{Supplementary Files}

This is a list of supplementary files associated with this preprint. Click to download.

- SupplementaryMaterials.pdf 